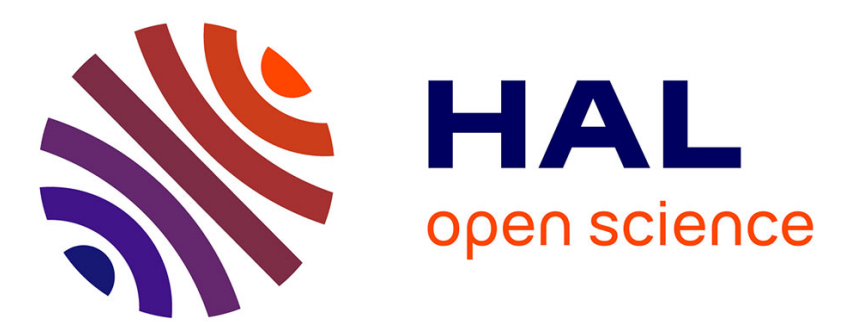

\title{
Discrete 3D model as complimentary numerical testing for anisotropic damage Arnaud Delaplace, Rodrigue Desmorat
}

\section{To cite this version:}

Arnaud Delaplace, Rodrigue Desmorat. Discrete 3D model as complimentary numerical testing for anisotropic damage. International Journal of Fracture, 2008, pp.20. 10.1007/s10704-008-9183-9 . hal-00270147

\section{HAL Id: hal-00270147 \\ https://hal.science/hal-00270147}

Submitted on 3 Apr 2008

HAL is a multi-disciplinary open access archive for the deposit and dissemination of scientific research documents, whether they are published or not. The documents may come from teaching and research institutions in France or abroad, or from public or private research centers.
L'archive ouverte pluridisciplinaire HAL, est destinée au dépôt et à la diffusion de documents scientifiques de niveau recherche, publiés ou non, émanant des établissements d'enseignement et de recherche français ou étrangers, des laboratoires publics ou privés. 


\title{
Discrete 3D model as complimentary numerical testing for anisotropic damage
}

\author{
A. Delaplace (delaplace@lmt.ens-cachan.fr) and R. Desmorat \\ (desmorat@lmt.ens-cachan.fr) \\ LMT-Cachan, ENS de Cachan / CNRS UMR 8535 / Université Paris 6 \\ 61, avenue du Président Wilson, 94235 Cachan, France
}

\begin{abstract}
It is proposed to use a discrete particle model as a complimentary "numerical testing machine" to identify the hydrostatic elasticity-damage coupling and the corresponding sensitivity to hydrostatic stresses parameter. Experimental tri-axial tensile testing is difficult to perform on concrete material, and numerical testing proves then its efficiency. The discrete model used for this purpose is based on a Voronoi assembly that naturally takes into account heterogeneity. Tri-tension tests on a cube specimen, based on a damage growth control, are presented. A successful identification of the hydrostatic sensitivity function of a phenomenological anisotropic damage model is obtained.
\end{abstract}

Keywords: discrete model, anisotropic, damage, cross-identification, virtual testing

\section{Introduction}

Experimental testing is an essential task when designing structures or when developing a constitutive model. Testing validates the accuracy of the structure design or the main model features. But experimental tests are usually limited due to their cost, and sometime to their complexity. In contrast with the past, tests number tends to decrease when planning a development campaign, and nonlinear numerical simulations are complimentary used (see (Linde et al., 2006; Reese, 2006; Wang et al., 2006) for some recent examples). In a near future numerical simulations may replace a non negligible part of experimental validation. If the model used is robust and efficient, simulations have numerous advantages: "perfect" boundaries and well-known loading conditions, limited cost, reproducibility.

The purpose here is to show that numerical simulations can help in the same way to identify parts of phenomenological constitutive models, as they can help to study bifurcation and instability (Delaplace et al., 1999). Material parameters identification is usually done with standard experimental tests (e.g. tension, compression, torsion) but which may be not sufficient for complex models or for models with a large number of parameters. This feature leads to the development of specific experimental devices and complex protocols in order to identify the "recalcitrant" or low sensitive parameters.

The phenomenological constitutive model considered in the present work is a 3D anisotropic damage model based on a reduced number of parameters (Desmorat, 2004; Desmorat et al., 2007). A function $h(\mathbf{D})$ controls the evolution of the damage $\mathbf{D}$ under positive hydrostatic stresses, and is tricky to determine for brittle heterogeneous material like mortar or ceramic: the response is highly sensitive to this function under triaxial states of stresses but becomes much less sensitive under uniaxial loading. It is proposed to use a discrete particle model (see (Cundall and Strack, 1979) for first application to geomaterials) well-adapted to describe material failure under tensile conditions, to perform pertinent complimentary numerical testing and to identify both the scalar argument of the function $h(\mathbf{D})$ (is it the norm of the damage tensor? the mean or hydrostatic damage?) and its expression. The 3D anisotropic damage and discrete particle models are described 
first, with a particular attention to parameters identification. A numerical identification protocol is given aiming at cross-identifying the discrete and phenomenological models damage hydrostatic responses.

\section{Induced anisotropic damage model}

The main idea of anisotropic damage models is to represent the non isotropic micro-cracks damage pattern. It is essential in 3D to built a state potential which can be continuously differentiated and from which derive the state laws: the elasticity law coupled with damage and the definition of the thermodynamics force associated with damage. This key differentiability feature ensures the stresses-strains continuity under complex non proportional loading. Anisotropic damage is generally represented by a tensorial thermodynamics variable D (Chaboche, 1978; Leckie and Onat, 1981; Cordebois and Sidoroff, 1982; Ladevèze, 1983; Chow and Wang, 1987; Murakami, 1988; Ju, 1989; Halm and Dragon, 1998; Lemaitre and Desmorat, 2005) taken next as a second order tensor. An anisotropic damage model for concrete has been proposed based on these assumptions (Desmorat, 2004; Desmorat et al., 2007), based also on a splitting of the Gibbs free enthalpy (Papa and Taliercio, 1996; Lemaitre et al., 2000)

- into a deviatoric part fully affected by the damage tensor $\mathbf{D}$ through the effective tensor $\mathbf{H}=(\mathbf{1}-\mathbf{D})^{-1 / 2}$,

- and on a hydrostatic part affected by a sensitivity to hydrostatic stresses scalar function $h(\mathbf{D})$ for positive hydrostatic stresses and not affected by damage for negative hydrostatic stresses.

Using the notation $\langle x\rangle=\max (x, 0)$ for the positive part of a scalar, Gibbs free enthalpy reads:

$$
\rho \psi^{*}=\frac{1+\nu}{2 E} \operatorname{tr}\left[\mathbf{H} \boldsymbol{\sigma}^{D} \mathbf{H} \boldsymbol{\sigma}^{D}\right]+\frac{1-2 \nu}{6 E}\left[h(\mathbf{D})\langle\operatorname{tr} \boldsymbol{\sigma}\rangle^{2}+\langle-\operatorname{tr} \boldsymbol{\sigma}\rangle^{2}\right]
$$

with $E$ and $\nu$ the Young modulus and Poisson ratio of the initially isotropic material and where $(\cdot)^{D}$ denotes the deviatoric part. The purpose next is to determine $h(\mathbf{D})$ function for concrete.

The state laws derive from the state potential (1), the elasticity law reading then

$$
\boldsymbol{\varepsilon}=\rho \frac{\partial \psi^{*}}{\partial \boldsymbol{\sigma}}=\frac{1+\nu}{E}\left[\mathbf{H} \boldsymbol{\sigma}^{D} \mathbf{H}\right]^{D}+\frac{1-2 \nu}{3 E}[h(\mathbf{D})\langle\operatorname{tr} \boldsymbol{\sigma}\rangle-\langle-\operatorname{tr} \boldsymbol{\sigma}\rangle] \mathbf{1}
$$

The strain energy release rate density - the thermodynamics force associated with the damage $\mathbf{D}$ - is gained as $\boldsymbol{Y}=\rho \frac{\partial \psi^{*}}{\partial \mathbf{D}}$.

Concerning damage, a criterion function $f$ is considered defining the elasticity domain by $f<0$ and damage growth by the consistency condition $f=0$ and $\dot{f}=0$,

$$
f=\hat{\varepsilon}-\kappa(\operatorname{tr} \mathbf{D})
$$

where $\hat{\varepsilon}$ is Mazars equivalent strain (Mazars, 1984; Mazars et al., 1990),

$$
\hat{\varepsilon}=\sqrt{\langle\varepsilon\rangle_{+}:\langle\varepsilon\rangle_{+}}
$$


built from the positive extensions $\left(\langle\varepsilon\rangle_{+}\right.$is the positive part of the strain tensor in terms of principal values). The function $\kappa$ allowing for modeling both tensile and compressive response of concrete with a single set of material parameters is:

$$
\kappa(\operatorname{tr} \mathbf{D})=a \cdot \tan \left[\frac{\operatorname{tr} \mathbf{D}}{a A}+\arctan \left(\frac{\kappa_{0}}{a}\right)\right]
$$

The anisotropic damage growth is assumed induced by the square of the positive strain tensor $^{1}\langle\varepsilon\rangle_{+}^{2}$ as ( $\dot{\lambda}$ is the damage multiplier gained from the consistency condition),

$$
\dot{\mathbf{D}}=\dot{\lambda}\langle\varepsilon\rangle_{+}^{2}
$$

The numerical implementation of the model in a finite element code is given in (Desmorat et al., 2007). The intrinsic dissipation due to damage remains positive (Desmorat, 2006). A main feature of the model is the reduced number of material parameters introduced to represent the full 3D anisotropic damage evolution: 5 including the elastic parameters,

- the elasticity parameters $E, \nu$,

- the damage threshold $\kappa_{0}$,

- the damage parameters $A, a$,

and the function $h(\mathbf{D})$.

The first five parameters are easily identified from basic experimental tension and compression tests. On the other hand, the function $h(\mathbf{D})$ is more subtle to identify, because it is acting on triaxial tension states difficult to represent with an experimental setup for brittle materials. An identification for metallic materials of such a function has been successfully realized (Lemaitre et al., 2000) but the procedure needed different small samples cut in large uniaxialy pre-damaged plates. The small samples were then tested to measure their damaged elastic properties related to $h$. The experimental protocol used is not conceivable for quasi-brittle material. With the fact that tests with positive hydrostatic stresses are very difficult to perform for these materials, numerical testing will prove useful in order to cross-identify the sensitivity to hydrostatic stresses function $h$.

The numerical tests will be made by use of a discrete model, based on a simple statistic representation of the material at the microstructure scale. Discrete modeling is robust enough in tension-like loadings to be considered as a numerical testing machine under such loading conditions (Kun and Herrmann, 1996; Bolander and Saito, 1998; D'Addetta et al., 2002; Yip et al., 2006; Delaplace and Ibrahimbegovic, 2006). Tri-tension loading tests can then be realized. Next section is devoted to the discrete model used.

\section{Discrete model as particle assembly}

In the considered discrete model, the material is described as a particles assembly (see the pioneering work of (Cundall and Strack, 1979)), representative of the material heterogeneity. Two kinds of particle shapes are generally used: spherical or polyhedra. The first one is very efficient thanks to the simple shape, especially for contact problems. But mesh

\footnotetext{
1 in terms of principal components, i.e. (i) make $\varepsilon$ diagonal as $\varepsilon_{\text {diag }}=\mathbf{P}^{-1} \varepsilon \mathbf{P}$, (ii) take its positive part $\left\langle\varepsilon_{\text {diag }}\right\rangle_{+}$, (iii) turn it back to the initial basis, $\langle\varepsilon\rangle_{+}=\mathbf{P}\left\langle\varepsilon_{\text {diag }}\right\rangle_{+} \mathbf{P}^{-1}$
} 
generation is tricky, and space between particles needs a special treatment for dynamic problems (see for example a full generation procedure in (Potyondy and Cundall, 2004)). On the other hand, control of heterogeneity and mesh generation are easy to obtain with polyhedra shapes. One chooses these last particles, computed from a Voronoi tessellation. Heterogeneity is controlled through the randomness of the particle center. There is no direct correlation between the Voronoi particles and the microstructure of a real material, but the introduced randomness avoids any privileged orientation in the medium. Because one wants a simple control of the boundary and loading conditions, a $3 \mathrm{D}$ regular grid is generated on the sample and a particle center is generated inside each grid box (Moukarzel and Herrmann, 1992). Figure 1 explains the successive steps of the mesh generation. A 2D mesh is considered for a better visualisation, but the steps remain identical in 3D.

1. Create a grid on the mesh outline and place random points in each square;

2. Compute the Delaunay triangulation of the set of points;

3. Compute the dual Voronoi tesselation;

4. Cut the particles with the mesh outline.
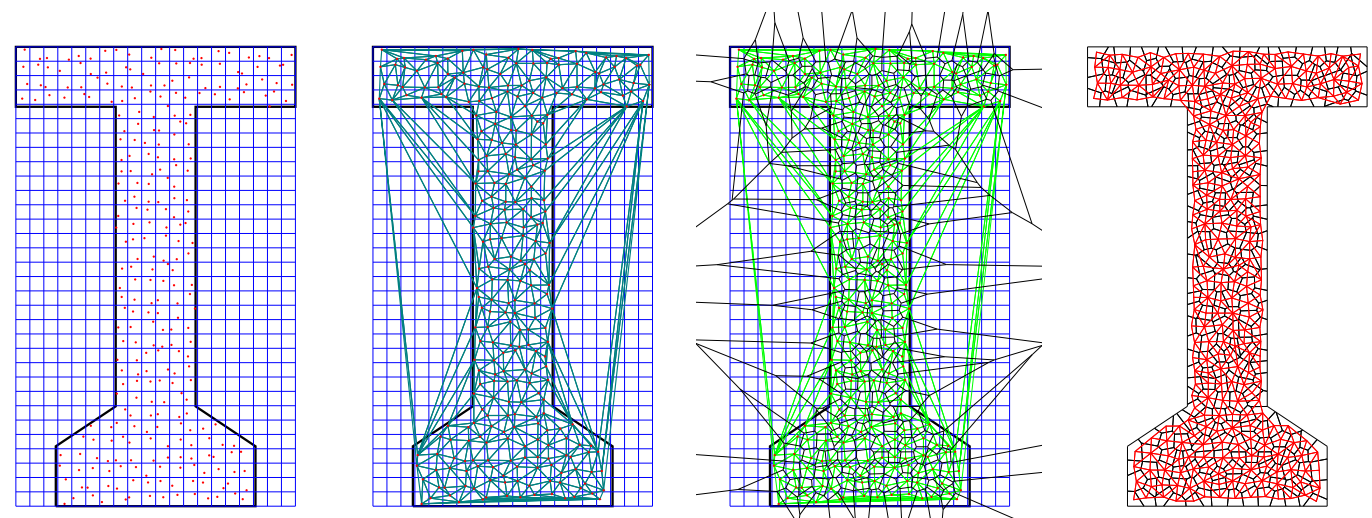

Figure 1. Successive steps of the mesh generation.

The geometry considered next is simpler (a cube) but 3D. Two different meshes are given in figure 2 .
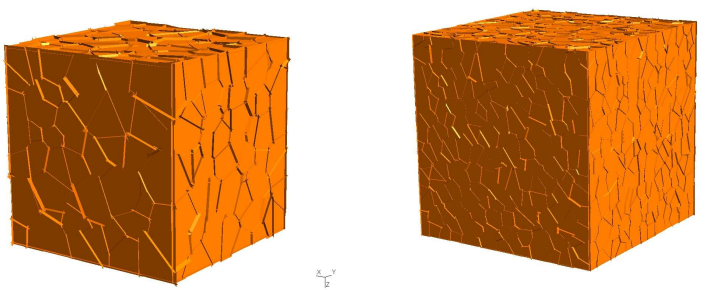

Figure 2. Two 3D samples used for this study (left: $8 \times 8 \times 8$, right: $16 \times 16 \times 16$ ). 


\subsection{Particle interactions}

Two kinds of interaction are taken into account, the cohesion forces and the contact forces. Cohesion forces are necessary in order to represent the behavior of a cohesive material, as contact forces are used for impact problems and for cyclic loading problems, to represent cracks closure effect. In this study, only tension and tritension tests will be considered. No contact forces are computed.

Because particles are underformable (an overlapping is allowed), particle interaction should represent the elastic material behavior. For two particles, the interaction is represented through a $12 \times 12$ local stiffness matrix. Generally, physical meaning of this matrix is rendered as six elastic springs at the particle common boundary, or as an elastic beam. This last representation is chosen and cohesive forces are represented by elastic EulerBernoulli beams. If just cohesive forces are considered, the model is nothing else than a lattice model (Schlangen and Garboczi, 1997; Van Mier et al., 2002).

An isotropic material is modeled here, characterized by two elastic parameters: $E$, the Young modulus, and $\nu$, the Poisson coefficient. These material parameters can be imposed by choosing the right local beam parameters. For an elastic Euler-Bernoulli beams, these parameters are:

- the Young modulus $E_{b}$ (equals for all beams),

- the area $A_{b}$,

- the length $\ell_{b}$,

- the moment of inertia $I_{b}$.

$A_{b}$ and $\ell_{b}$ are imposed by the particle geometry. Then, elastic material parameters $E$ and $\nu$ are obtained through the beam Young modulus $E_{b}$ and through the beam inertia. An adimensional parameter $\alpha=64 I_{b} /\left(\pi \phi^{4}\right)$ is introduced instead of $I_{b}$, with $\phi$ the diameter of the equivalent circular section of the considered beam.

With a discrete model, a "sufficient number" of particles should be considered in order to obtain convergence of the elastic properties of the media to the isotropic elastic properties of the material. This convergence is shown on figure 3 , for the following model parameters $E_{b}=46 \mathrm{GPa}$ and $\alpha=0.74$. For different particle densities and for different meshes, the macroscopic elastic properties $E$ and $\nu$ of the media have been computed. Table I summarizes the results. As expected, parameters converge toward a finite values as the density increases.

\subsection{NONLinEar BeHAVior}

Quasi-brittle response of the material is obtained by considering a brittle behavior of the beams. The breaking criteria depend on the beam axial strain and on the rotations of extremities $i$ and $j$ leading to the following expression:

$$
P_{i j}\left(\varepsilon_{i j},\left|\theta_{i}-\theta_{j}\right|\right) \geq 1
$$

where $\varepsilon_{i j}$ is the beam strain, $\theta_{i}$ and $\theta_{j}$ are respectively the rotations of the end particles $i$ and $j$ and where $P($.$) is a coupling function. If condition (7) is fulfilled, the beam breaks$ irreversibly. Enhanced behavior can be considered, with for instance linear softening, but 
Table I. Elastic parameters for different particle densities.

\begin{tabular}{|l|c|c|c|}
\hline $\begin{array}{l}\text { particle } \\
\text { density }\end{array}$ & $\begin{array}{c}\text { number of } \\
\text { realizations }\end{array}$ & $E(\mathrm{GPa})($ min-max) & $\nu$ (min-max) \\
\hline 4 & 2048 & $43.14(35.91-50.69)$ & $0.1622(0.08978-0.2450)$ \\
\hline 8 & 512 & $35.19(33.74-36.96)$ & $0.1881(0.1689-2.146)$ \\
\hline 16 & 256 & $31.96(31.62-32.36)$ & $0.1989(0.1904-0.2064)$ \\
\hline 24 & 128 & $31.00(30.80-31.17)$ & $0.2020(0.1974-0.2054)$ \\
\hline 32 & 64 & $30.56(30.46-30.67)$ & $0.2034(0.2014-0.2055)$ \\
\hline 40 & 8 & $30.32(30.28-30.37)$ & $0.2040(0.2032-0.2046)$ \\
\hline
\end{tabular}
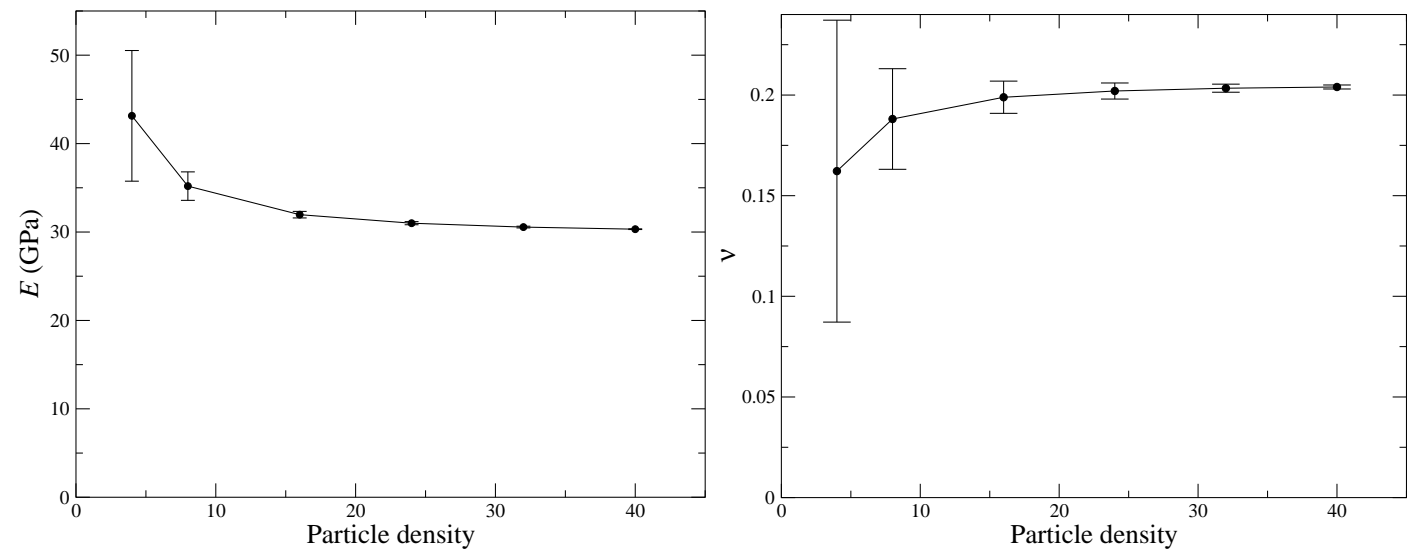

Figure 3. Convergence of elastic parameters with respect to particle density

computational cost increases much with the improvement. For brittle materials like concrete, brittle elastic behavior usually gives good results (Van Mier and Van Vliet, 2003). Following (Herrmann and Roux, 1990; D'Addetta, 2004), the chosen breaking threshold is:

$$
P_{i j}=\left(\frac{\varepsilon_{i j}}{\varepsilon_{c r}}\right)^{2}+\left(\frac{\left|\theta_{i}-\theta_{j}\right|}{\theta_{c r}}\right) \geq 1
$$

where the first variable $\varepsilon_{c r}$ acts mainly on tensile behavior as the second one $\theta_{c r}$ acts on compressive behavior.

\subsection{SOLVER}

We present in this part the algorithm used for static problems. Basically, one has to solve the discrete equilibrium equations, formally written

$$
\mathbf{K}(\mathbf{u}) \mathbf{u}=\mathbf{f}
$$

$\mathbf{K}(\mathbf{u})$ is the global stiffness matrix, $\mathbf{u}$ the displacement vector, $\mathbf{f}$ the loading vector applied to particles. The most common algorithm, also used in finite element codes, is the step- 
by-step monotonic loading algorithm, as follows for step $k$ corresponding to the applied $\operatorname{load} \mathbf{f}^{k}$ :

Step $k$

1. apply loading $\mathbf{f}^{k}$,

2. compute $\mathbf{u}^{k}$ using an iterative method solving equation (9),

3. save couple $\left(\mathbf{u}^{k}, \mathbf{f}^{k}\right)$,

4. find the $m_{k}$ links that satisfy

$$
P_{i_{p} j_{p}} \geq 1 \quad p \in\left\{1, . ., m_{k}\right\}
$$

5. change the stiffness matrix setting

$$
\mathbf{K}^{k+1}=\mathbf{K}^{k}-\sum_{p=1}^{m_{k}} \mathbf{L}_{i_{p} j_{p}}^{T} \mathbf{K}_{i_{p} j_{p}} \mathbf{L}_{i_{p} j_{p}}
$$

where $\mathbf{L}_{i_{p} j_{p}}$ is the connectivity matrix of element $i_{p} j_{p}$.

End step $k$

The drawback with this algorithm is that the response depends on the loading step $\Delta \mathbf{f}=\mathbf{f}^{k+1}-\mathbf{f}^{k}$. Furthermore, if the loading step is too large, the algorithm may not converge. Then, one prefers a second algorithm, called the elastic prediction algorithm which ensures a unique response. Global loading does not correspond to a monotonic increasing force or increasing displacement, but corresponds to a decreasing of the apparent stiffness. Usually, just one beam is broken during one step. The algorithm is:

Step $k$

1. apply elastic loading $\mathbf{f}^{e l}$,

2. compute $\mathbf{u}^{\text {el }}$ using an iterative method solving equation (9),

3. compute $\theta_{\min }$ with

$$
\theta_{\min }=\min _{\substack{i, j \in(1, . ., n) \\ i \neq j}}\left(\frac{1}{P_{i j}}\right)
$$

4. save couple $\left(\theta_{\min } \mathbf{u}^{e l}, \theta_{\min } \mathbf{f}^{e l}\right)$,

5. change the stiffness matrix setting

$$
\mathbf{K}^{k+1}=\mathbf{K}^{k}-\mathbf{L}_{i j}^{T} \mathbf{K}_{i j} \mathbf{L}_{i j}
$$

where $\mathbf{L}_{i j}$ is the connectivity matrix of element $i j$.

End step $k$

Note that such an algorithm can also be used in a finite element code (Rots et al., 2006). The two algorithms give the same response for stable crack propagation, and for a sufficient small loading step for the monotonic algorithm. The response force-displacement obtained 
with the monotonic algorithm is nothing else than the envelope of the response obtained with the elastic prediction algorithm. In the following, we will use this last algorithm to avoid loading step dependency.

\subsection{Discrete model Parameter identification}

\subsubsection{Elastic parameters}

The model elastic parameters are $E_{b}$, the beam Young modulus, and $\alpha$, the inertia coefficient. These two parameters are identified by considering the isotropic elastic material coefficients $E$, the Young modulus, and $\nu$, the Poisson coefficient of the macroscopic medium. This identification is easy when considering these two following properties:

- the material Young modulus $E$ is proportional to beam Young modulus $E_{b}$.

- the material Poisson coefficient $\nu$ does not depend on the beam Young modulus.

These properties are shown on figure 4 . Evolution and $E$ and $\nu$ are plotted versus $E_{p}$ with a fixed $\alpha$, and versus $\alpha$ with a fixed $E_{b}$.
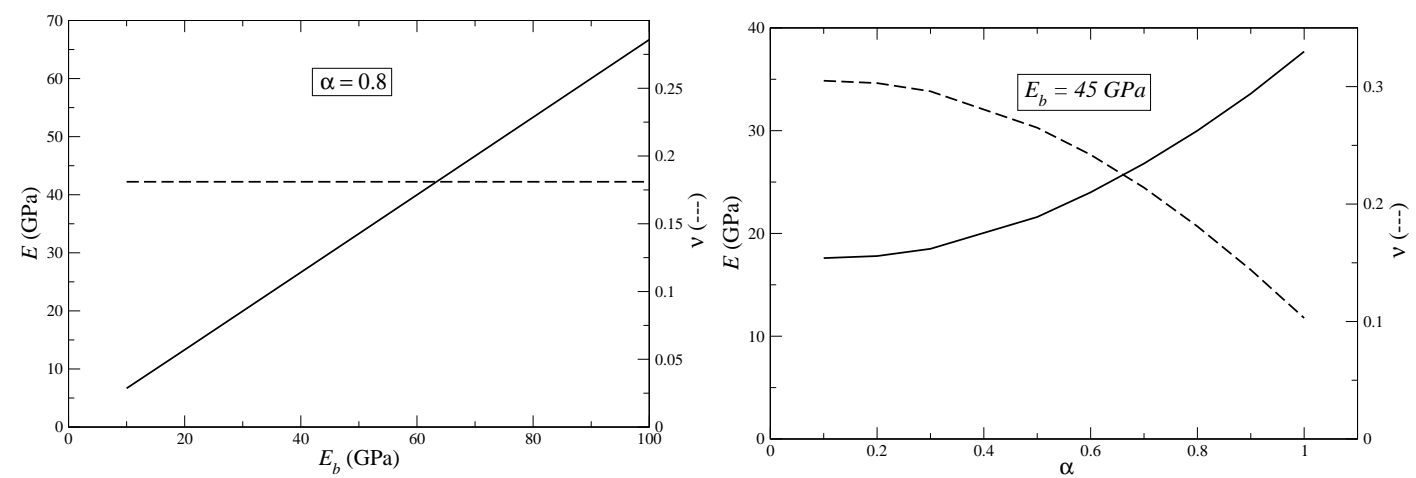

Figure 4. Evolution of elastic material parameters $E$ and $\nu$ versus beam parameters $E_{b}$ and $\alpha$.

Then, the identification proceeds in two steps:

1. Calibrate $\alpha$ with respect to material Poisson coefficient (eventually by using figure 4 , right)

2. Calibrate $E_{b}$ with respect to material Young coefficient (eventually by using figure 4 , left)

\subsubsection{Nonlinear parameters}

For the identification of the nonlinear parameters, one has to keep in mind that rupture of quasi-brittle materials are mainly due to apparition of mode-I microcracks. Two variables, $\varepsilon_{c r}$ and $\theta_{c r}$, control the nonlinear behavior for the chosen model. One identifies these two variables on the peak stress values in tension and in compression. As for the elastic parameters, identification of $\varepsilon_{c r}$ and $\theta_{c r}$ proceeds in two steps. The tension peak stress value depends indeed only on $\varepsilon_{c r}$ : a simple tension test allows to identify $\varepsilon_{c r}$ value. Then, a simple compression loading is used to identify $\theta_{c r}$ value with respect to the peak stress.

Figure 5 shows the evolution of the tension peak load versus $\varepsilon_{c r}$, for a fixed $\theta_{c r}$, and versus $\theta_{c r}$, for a fixed $\varepsilon_{c r}$. As expected, the peak stress depends mainly on $\varepsilon_{c r}$ with a linear 
relationship. Note that a dependence with respect to $\theta_{c r}$ exists for small values, but these values have no physical meaning for the modeled material.
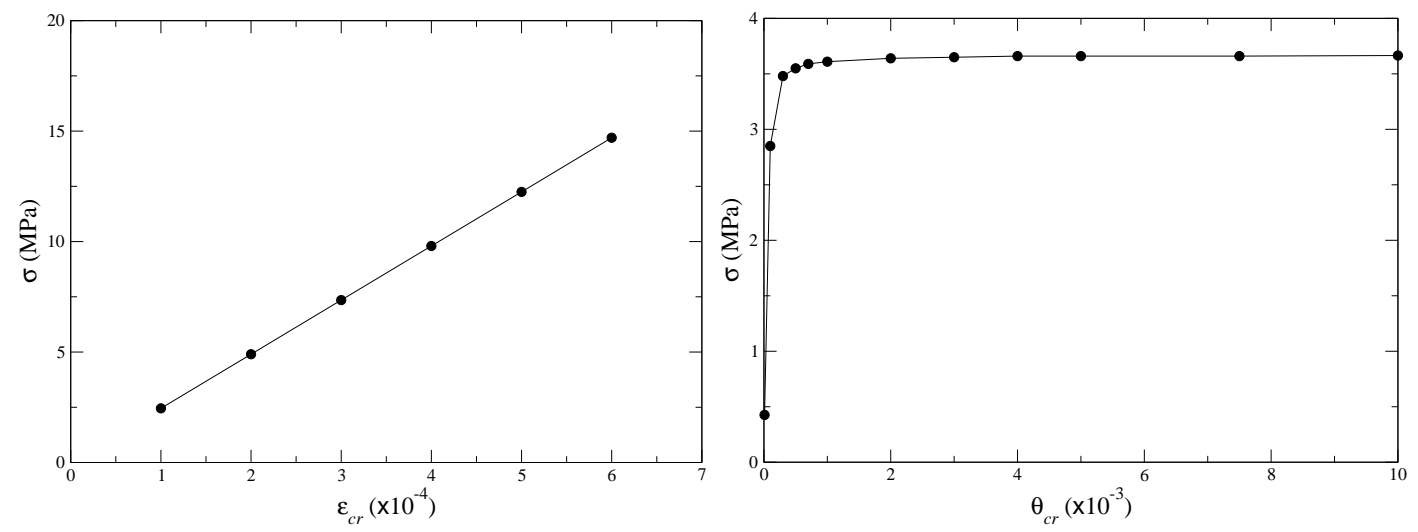

Figure 5. Evolution of tension peak stress versus model parameters $\varepsilon_{c r}$ and $\theta_{c r}$.

As mentioned earlier, the second step consists in the evaluation of $\theta_{c r}$ from a simple compression test. The relationship between peak stress and $\theta_{c r}$ is not linear, and identifying this last variable is obtained using figure 6 , where the evolution of the compression peak stress is plotted versus $\theta_{c r}$.

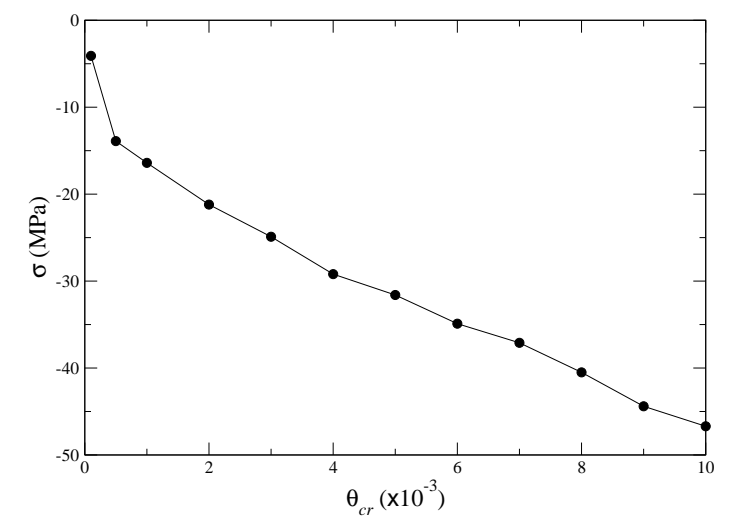

Figure 6. Evolution of compression peak stress versus mean model parameter $\theta_{c r}$.

Finally, the identified model parameters for a tension peak stress of $3 \mathrm{MPa}$ and a compression peak stress of $-30 \mathrm{MPa}$ are:

$$
\varepsilon_{c r}=1.8 \times 10^{-4}, \quad \theta_{c r}=5.6 \times 10^{-3}
$$

The response of the discrete model for these last values is plotted in figure 7, for either tension or compression.

\section{Cross-identification of function $h(\mathbf{D})$}

A strategy for determining the sensitivity to hydrostatic stresses function $h$ is developed by using different relations gained from the elasticity coupled with damage law (2). Whatever 


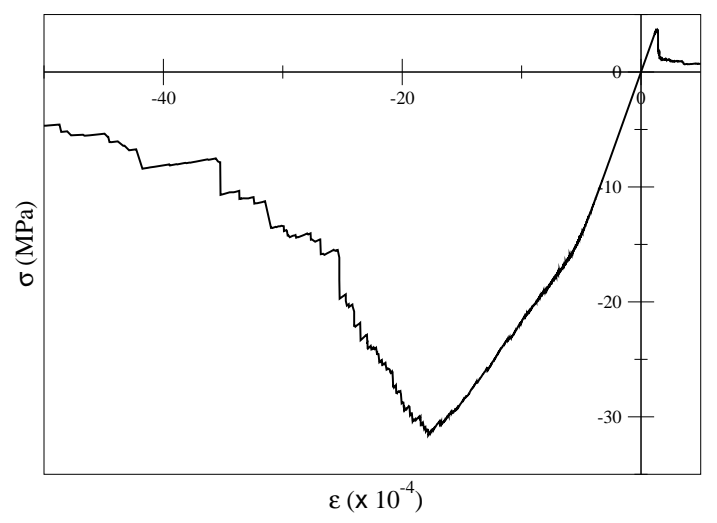

Figure 7. Discrete model response for parameters $\varepsilon_{c r}=1.8 \times 10^{-4}$ and $\theta_{c r}=5.6 \times 10^{-4}$.

the chosen strategy, a tritension loading test has to be performed with a bulk modulus evaluation for different damage values, test excessively difficult to realize experimentally.

\subsection{IDENTIFICATION PROCEDURE}

The identification strategy is based on the expression of the damaged - or effective - bulk modulus:

$$
\tilde{K}=\frac{\operatorname{tr} \sigma}{3 \operatorname{tr} \varepsilon}
$$

By using expression (2), one has:

$$
\tilde{K}=\frac{K}{h(\mathbf{D})}
$$

where $K$ is the modulus of the virgin material. One can propose the following global identification procedure. Note that the term "measure" (or measurement) means "measure on the computed response by means of the discrete modeling".

- Perform one elastic uniaxial tension test on a cube.

- Measure $E, \nu$ and the initial bulk modulus $K=E / 3(1-2 \nu)$

- Perform n-nonlinear tritension tests using the discrete model for different increasing loads $q \in\{1, . . n\}$.

Proceeds as follows:

1. Apply equally imposed displacements $\bar{u}_{1}^{q}=\bar{u}_{2}^{q}=\bar{u}_{3}^{q}$ on the cube faces and measure the damaged bulk modulus $\tilde{K}^{q}$.

2. Unload the specimen.

3. Apply an elastic uniaxial load in direction $i$.

- Measure the damaged Young modulus $\tilde{E}_{i}^{q}$

- Compute the damage value as (obtaining of next expression is detailed in appendix A)

$$
D^{q}=1-\frac{6(1+\nu) \tilde{E}^{q} \tilde{K}^{q}}{E\left(9 \tilde{K}^{q}-\tilde{E}_{i}^{q}\right)}
$$


- Store the couple $\left(\tilde{K}^{q}, D^{q}\right)$

- Identify $h$ from the curve $K / \tilde{K}^{q}=h\left(\mathbf{D}^{q}\right), q \in\{1, . . n\}$.

For this last point two assumptions will be compared: a) $h=h\left(D_{H}\right)$ with $D_{H}=\frac{1}{3} \operatorname{tr} \mathbf{D}$ the hydrostatic damage and b) $h=h(\|\mathbf{D}\|)$ with $\|\mathbf{D}\|=\sqrt{\mathbf{D}: \mathbf{D}}$ the norm of tensor D.

Note that if the iso-triaxial damage assumption is not satisfied, the two last points of the protocol are changed into:

- Perform n-nonlinear tritension tests using the discrete model for different loads $q \in$ $\{1, . . n\}$.

Proceeds as follows:

1. Apply equally imposed displacements $\bar{u}_{1}^{q}=\bar{u}_{2}^{q}=\bar{u}_{3}^{q}$ on the cube faces and measure the damaged bulk modulus $\tilde{K}^{q}$.

2. Unload the specimen.

3. Apply three elastic uniaxial loads in the three loading directions $x \equiv 1, y \equiv 2, z \equiv$ 3 .

- Measure damaged Young modulus $\tilde{E}_{1}^{q}, \tilde{E}_{2}^{q}$ and $\tilde{E}_{3}^{q}$

- Compute damage values for each direction (obtaining of next expressions is detailed in appendix B)

$$
\begin{gathered}
1-D_{1}^{q}=\frac{2(1+\nu)}{E\left(\frac{5}{\tilde{E}_{1}^{q}}-\frac{1}{\tilde{E}_{2}^{q}}-\frac{1}{\tilde{E}_{3}^{q}}-\frac{1}{3 \tilde{K}^{q}}\right)}, \\
1-D_{2}^{q}=\frac{2(1+\nu)}{E\left(-\frac{1}{\tilde{E}_{1}^{q}}+\frac{5}{\tilde{E}_{2}^{q}}-\frac{1}{\tilde{E}_{3}^{q}}-\frac{1}{3 \tilde{K}^{q}}\right)}, \\
1-D_{3}^{q}=\frac{2(1+\nu)}{E\left(-\frac{1}{\tilde{E}_{1}^{q}}-\frac{1}{\tilde{E}_{2}^{q}}+\frac{5}{\tilde{E}_{3}^{q}}-\frac{1}{3 \tilde{K}^{q}}\right)},
\end{gathered}
$$

- $\quad$ Store the set $\left(\tilde{K}^{q}, D_{1}^{q}, D_{2}^{q}, D_{3}^{q}\right)$

- Identify $h$ from the curve $K / \tilde{K}^{q}=h\left(\mathbf{D}^{q}\right), q \in\{1, . . n\}$ with either assumption a) or b).

\subsection{Numerical Results}

The identification of function $h(\mathbf{D})$ is performed on two cube samples (also illustrated in figure 13). Two different cube sizes are considered, the increase in size corresponding to an increase in the number of particles and in the number of degrees of freedom (dof). The discrete model parameters are $E_{b}=45 \mathrm{GPa}, \alpha=0.75, \varepsilon_{c r}=1.8 \times 10^{-4}, \theta_{c r}=5.0 \times 10^{-3}$. 
Table II. Samples tested for the determination of function $h(\mathbf{D})$.

\begin{tabular}{|c|c|c|}
\hline sample size & number of particles & number of dof \\
\hline $8 \times 8 \times 8$ & 512 & 3072 \\
\hline $16 \times 16 \times 16$ & 4096 & 24576 \\
\hline
\end{tabular}

\subsubsection{8-cube sample results}

Tritension response of the 8-cube sample is plotted in figure 8 (for the crack pattern see directly figure 13). Stress-strain curves are plotted for the three directions of loading and, more important, evolution of the damage moduli $\tilde{E}_{i}, i \in\{1,2,3\}$ are the right-hand curves (upperscripts $q$ corresponding to the maximum applied displacement are omitted next). From these values, the bulk modulus $\tilde{K}$ is computed. Recall that $\operatorname{tr}(\mathbf{D})=3 D_{H}=$ $D_{1}+D_{2}+D_{3}$ and $\|\mathbf{D}\|=\sqrt{D_{1}^{2}+D_{2}^{2}+D_{3}^{2}}$ are respectively the hydrostatic damage and the norm of the damage tensor in the principal framework. Note that $\operatorname{tr}(\mathbf{D})$ and $\|\mathbf{D}\|$ are both equal in homogeneous uniaxial tension as then $\operatorname{tr}(\mathbf{D})=\|\mathbf{D}\|=D_{1}$ (in direction 1).
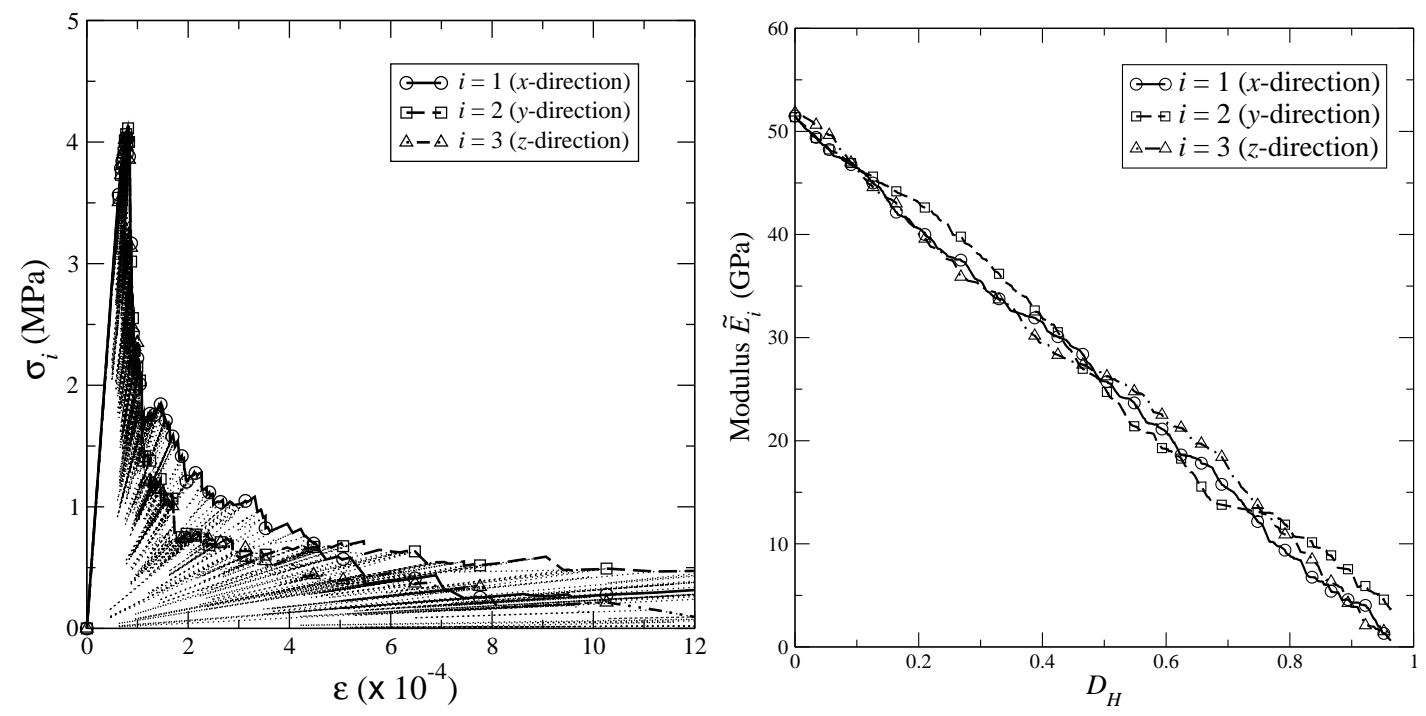

Figure 8. Stress-strain responses for the three directions during tri-tension (left), and evolution of stiffnesses $\tilde{E}_{1}, \tilde{E}_{2}, \tilde{E}_{3}$ (right).

For successive loading steps, tritension test is stopped and an uniaxial tensile loading is applied elastically in order to obtain the corresponding principal damage values $D_{i}$. Finally, the evolution of the ratio $\tilde{K} / K$, i.e. the inverse of function $h(\mathbf{D})$, is plotted in figure 9 . The left-hand side figure shows this evolution versus $D_{H}$ (assumption a), and the righthand side one versus the $\|\mathbf{D}\|$ (assumption b). In order to reveal the intrinsic property of function $h(\mathbf{D})$, curves obtained for uniaxial tension tests in the different directions $x \equiv 1$, $y \equiv 2, z \equiv 3$ (instead of triaxial tension) are superimposed. One can see that $h\left(D_{H}\right)$ is kept invariant when $h(\|\mathbf{D}\|)$ depends on the loading state. This result justifies the choice 
$h=h\left(D_{H}\right)$ for the damage coupling in the hydrostatic part of Gibbs thermodynamics potential rather than the choice $h=h(\|\mathbf{D}\|)$.
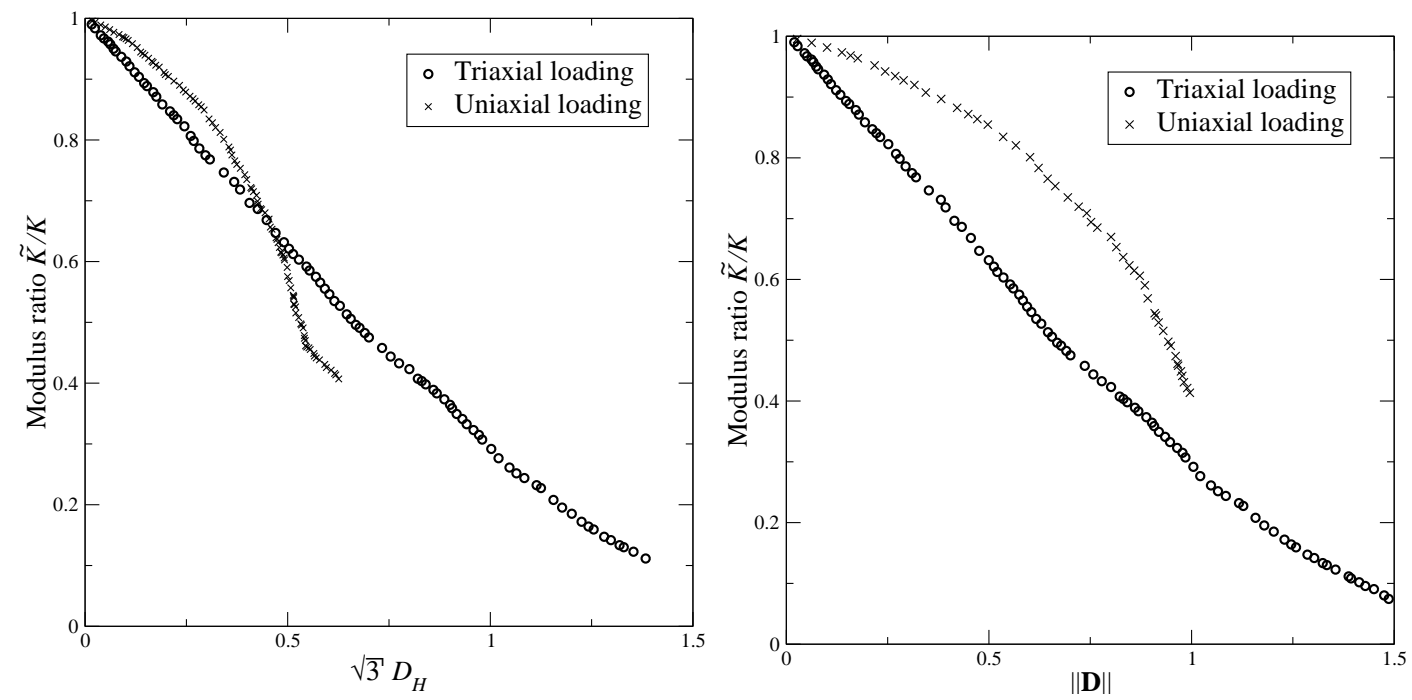

Figure 9. Evolution of $\tilde{K} / K=1 / h(\mathbf{D})$ versus hydrostatic damage (left, in fact versus $\sqrt{3} D_{H}$ for comparison) and versus the norm $\|\mathbf{D}\|$ (right) for the 8-cube sample: $h(\|\mathbf{D}\|)$ exhibits a loading dependency when $h\left(D_{H}\right)$ is kept invariant and can be considered as intrinsic.

The second main conclusion concerns the final determination of function $h$. A linear evolution of $\tilde{K} / K$ is obtained over a wide range of damage values. Hence, $1 / h(\mathbf{D})$ can be considered as an affine function of $D_{H}$ characterized by a slope $\eta$, leading to:

$$
h(\mathbf{D})=\frac{1}{1-\eta D_{H}}
$$

Six samples have been broken in tritension for the identification of $\eta$. Evolution of $\tilde{K} / K=$ $1 / h(\mathbf{D})$ versus hydrostatic damage $D_{H}$ for the six samples is shown in figure 10 . Parameter $\eta$ is evaluated from the best fitted line as

$$
\eta \approx 1.3
$$

The form(13) of function $h$ is in agreement with results obtained heuristicaly for metallic materials (Lemaitre et al., 2000), with only a different value for parameter $\eta$ (1.3 instead of values ranging between 2 and 3). This relationship emphasizes the fact that $\eta$ can be considered as a material parameter: the hydrostatic stresses sensitivity parameter.

Note that the isotropic damage assumption is satisfied for this sample (equality $D_{1}=$ $D_{2}=D_{3}$ during loading).

\subsubsection{6-cube sample results}

Figure 11 shows the results for the 16-cube sample. As for the 8-cube sample, $h\left(D_{H}\right)$ is kept invariant with respect to the loading conditions, but not $h(\|\mathbf{D}\|)$. Identification of parameter $\eta$ (eq. 13) is obtained from figure 12 in which bulk modulus measurements on uniaxialy damaged specimens are superimposed.

Note that localization occurs for the $y \equiv 2$ and $z \equiv 3$ directions after the peak load making the specimen a full structure instead of an equivalent Gauss point. One has to 


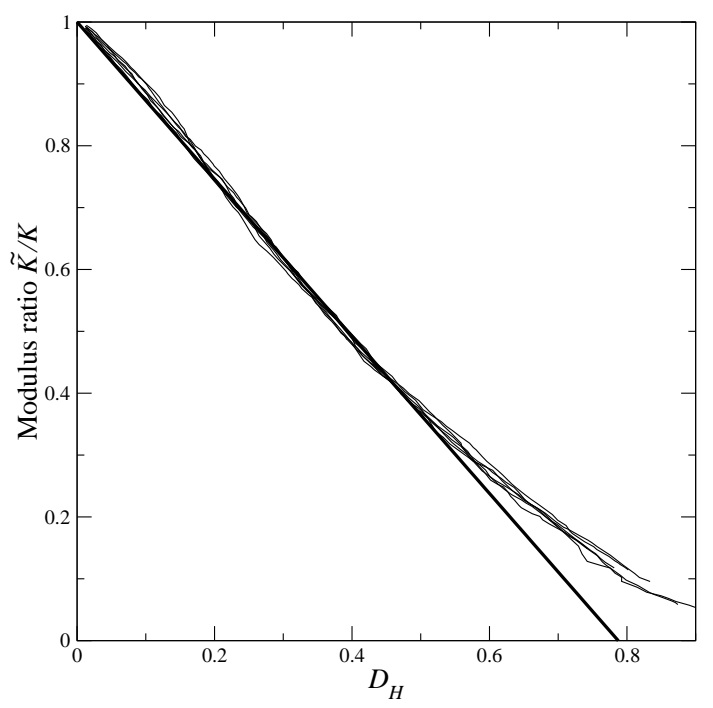

Figure 10. Evolution of $\tilde{K} / K=1 / h(\mathbf{D})$ versus hydrostatic damage $D_{H}$ for the six 8-cube samples. Straight line corresponds to $\tilde{K} / K=1-\eta D_{H}$.

limit the identification of the damage hydrostatic parameter to the beginning of the loading (prior to localization) if the iso-triaxial damage assumption is used. Parameter $\eta$ is evaluated to be:

$$
\eta \approx 1.2
$$
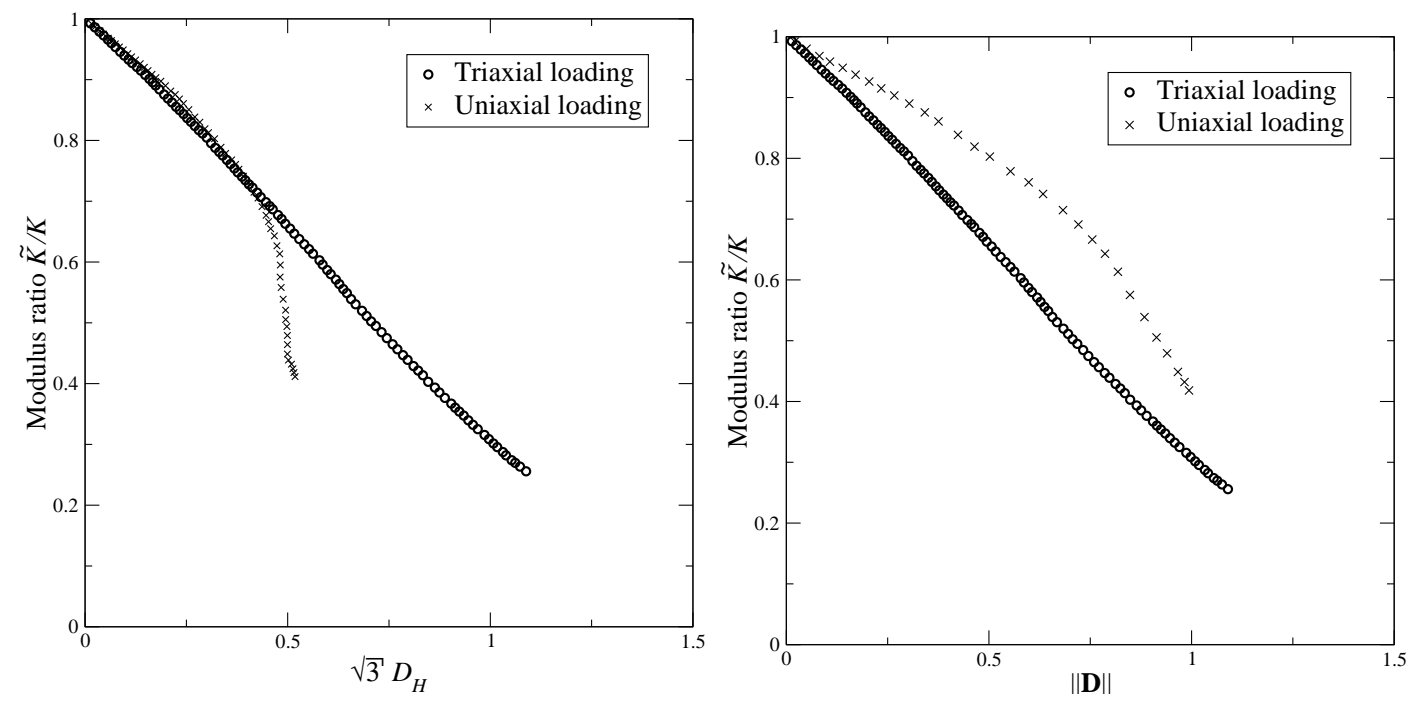

Figure 11. Evolution of $\tilde{K} / K=1 / h(\mathbf{D})$ versus hydrostatic damage (left, in fact versus $\sqrt{3} D_{H}$ for comparison) and versus the norm $\|\mathbf{D}\|$ (right) for the 16-cube sample.

The crack patterns obtained for the two samples are shown in figure 13. Note that the number of beams to break before failure varies from 1500 beams for the 8-cube sample to 8000 for the 16 one. Using elastic prediction algorithm needs to solves 8000 systems of 24576 degrees of freedom. 


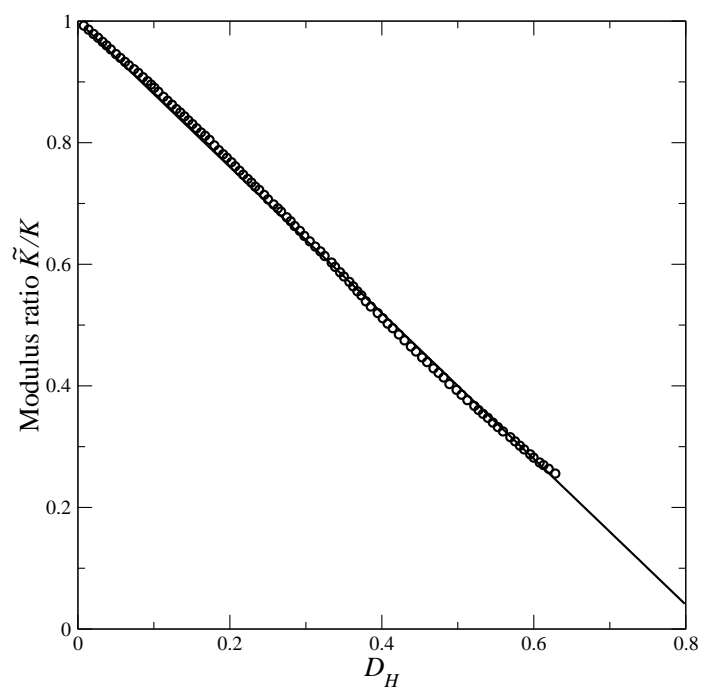

Figure 12. Evolution of $\tilde{K} / K=1 / h(\mathbf{D})$ versus hydrostatic damage $D_{H}$ for the 16 -cube sample. Straight line corresponds to $\tilde{K} / K=1-\eta D_{H}$.
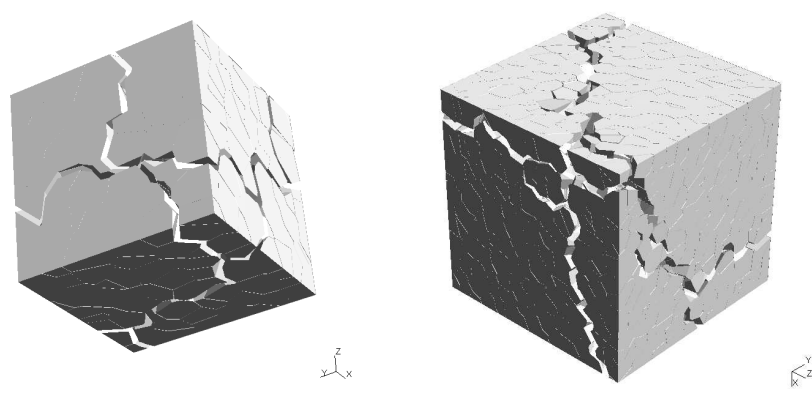

Figure 13. Crack patterns for the two samples (left $8 \times 8 \times 8$, right $16 \times 16 \times 16$ ).

\section{Identification of the anisotropic damage model}

One can now represent the response of the anisotropic damage model with the identified sensitivity to hydrostatic stresses function $h(\mathbf{D})=1 /\left(1-\eta D_{H}\right)$, the elasticity law reading:

$$
\boldsymbol{\varepsilon}=\frac{1+\nu}{E}\left[(\mathbf{1}-\mathbf{D})^{-1 / 2} \boldsymbol{\sigma}^{D}(\mathbf{1}-\mathbf{D})^{-1 / 2}\right]^{D}+\frac{1-2 \nu}{3 E}\left[\frac{\langle\operatorname{tr} \boldsymbol{\sigma}\rangle}{1-\frac{\eta}{3} \operatorname{tr} \mathbf{D}}-\langle-\operatorname{tr} \boldsymbol{\sigma}\rangle\right] \mathbf{1}
$$

The monotonic response in tension and compression is plotted in figure 14. On this curve, the effect of parameter $\eta$ cannot be noticed (it does not affect compression). The sensitivity to $\eta$ is shown in figure 15 , with as different values considered $\eta=0, \eta=1.25, \eta=3$. As expected, the response in uniaxial tension is not much influenced by this parameter. On the other hand, tritension response strongly depends of $\eta$. Note that the value $\eta=0$ corresponds to unphysical response with no damage developed in tritension. 


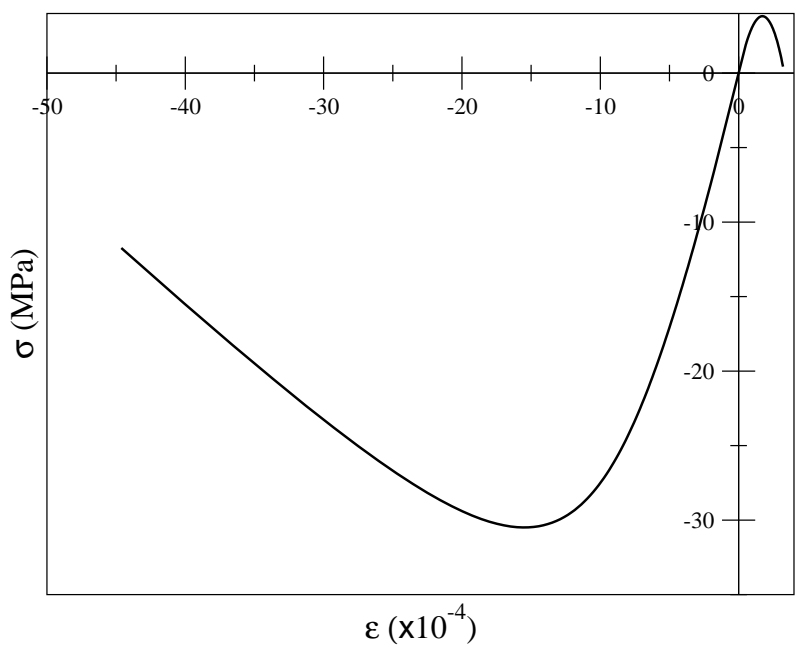

Figure 14. Response of the model in tension and compression $\left(E=37 \mathrm{GPa}, \nu=0.2, \kappa_{0}=5 \times 10^{-5}\right.$, $a=3 \times 10^{-4}, A=5 \times 10^{3}$ and $\left.\eta=1.25\right)$.

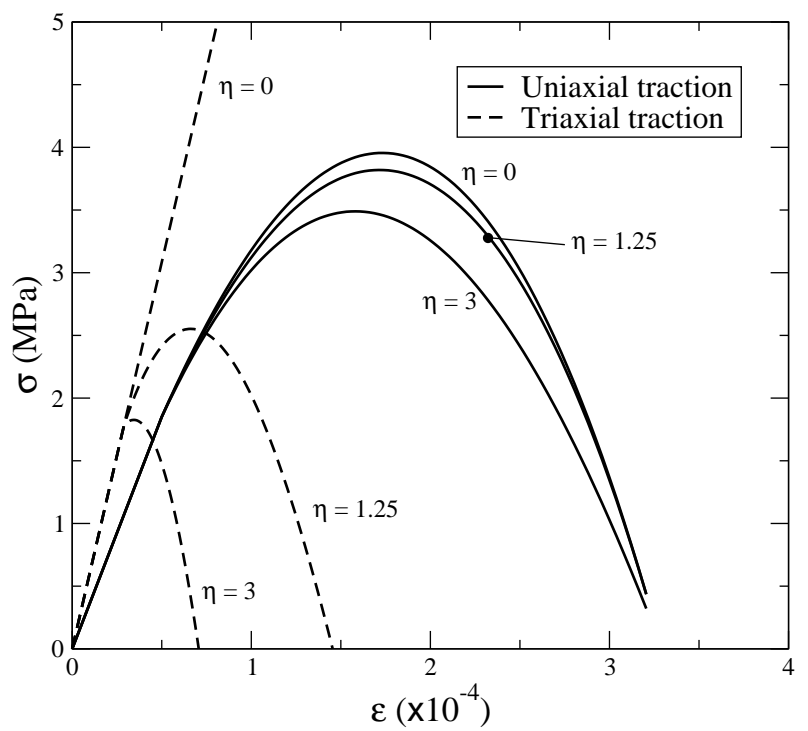

Figure 15. Effect of parameter $\eta$ for uniaxial (continuous line) and triaxial (dashed line) tension tests $\left(E=37 \mathrm{GPa}, \nu=0.2, \kappa_{0}=5 \times 10^{-5}, a=3 \times 10^{-4}\right.$ and $\left.A=5 \times 10^{3}\right)$.

\section{Conclusion}

The popularity and the use of a constitutive model depend on its robustness, its simplicity, and its easiness to implement in a numerical code. Concerning simplicity, the number, the physical meaning and the identification easiness of material parameters is an important feature to be considered. Both the discrete and anisotropic damage models have been developed with respect to these considerations with quite a reduced number of parameters introduced.

The experiments needed to identify the coupling - through a function $h$ - between positive hydrostatic stresses and anisotropic damage have been advantageously replaced 
by numerical testing and models cross-identification. A 3D discrete particle analysis has allowed us to determine the sensitivity to hydrostatic function $h(\mathbf{D})$ as an intrinsic function of the hydrostatic damage $D_{H}$,

$$
h(\mathbf{D})=h\left(D_{H}\right)=\frac{1}{1-\eta D_{H}}
$$

The value of the sensitivity to hydrostatic stresses parameter has also been determined for quasi-brittle materials,

$$
\eta \approx 1.25
$$

and is then quite different from the values obtained for metals for which $\eta \in[2,3]$.

To conclude, the advantages of numerical testing approach are numerous and have proven efficient:

- All tensile tests (uniaxial, biaxial, triaxial) can easily be considered in discrete modeling when the application of the corresponding loading conditions are most delicate in experiments. One performed a tri-tension loading on a cube sample without developing a specific setup device.

- Different loading paths can be realized on the same sample. This point is very important for brittle heterogeneous materials for which response variability is observed for different samples. In our case, one has performed 3 uniaxial tensile loadings in the 3 space directions on the same triaxially damaged specimen, experiment that could not be envisaged on a real specimen.

- The procedure for parameter identification is not restricted by the experimental setup. Then, the most suitable and robust procedure can be used, rather than an identification based on a difficult experimental test with then possibly ill-defined boundary conditions.

As a final remark, let us emphasize that numerical identification is obviously a complimentary procedure for experimental testing, and one does not imagine a full model identification with just numerical tests. But again this approach is an excellent possibility to identify specific material parameters.

\section{Appendix}

\section{A. Damage measurement under iso-damage assumption}

This appendix details the computation of the damage parameter of a specimen under an uniaxial elastic tensile loading. Before this loading, the specimen has been damaged under a tritension test, with the iso-damage assumption, i.e. $D_{1}=D_{2}=D_{3}=\frac{1}{3} \operatorname{tr} \mathbf{D}=D_{H}$. In the following, tensile load is supposed to be applied in the $x$-direction. Strain is computed from relation (2):

$$
\varepsilon_{11}=\frac{2}{3} \frac{1+\nu}{E\left(1-D_{H}\right)} \sigma_{11}+\frac{1-2 \nu}{3 E} \sigma_{11} h(\mathbf{D})
$$


Note that if $D_{H}=0$, the elastic relation $\varepsilon_{11}=\sigma_{11} / E$ is recovered (with the initial value $h(\mathbf{0})=1$ for the virgin material). By using the expressions $K=E /(3(1-2 \nu))$ for the elastic bulk modulus and $\tilde{K}=K / h(\mathbf{D})$ for the damaged bulk modulus,

$$
\varepsilon_{11}=\left(\frac{2}{3} \frac{1+\nu}{E\left(1-D_{H}\right)}+\frac{1}{9 \tilde{K}}\right) \sigma_{11}
$$

The definition of the damaged Young modulus $\tilde{E}_{11}=\sigma_{11} / \varepsilon_{11}$ altogether with equation (16) lead to the final relation:

$$
1-D_{1}=\frac{6 \tilde{K} \tilde{E}(1+\nu)}{E(9 \tilde{K}-\tilde{E})}
$$

\section{B. Damage measurement under anisotropic state}

This appendix details the computation of the damage variable of a specimen submitted to an uniaxial elastic tensile load. Recall that before this loading, the specimen has been damaged under a tritension test, with different damage values $D_{1} \neq D_{2} \neq D_{3}$. In the following, tensile load is supposed to be applied in the $x$-direction. The strain in $x$-direction is derived from relation (2):

$$
\varepsilon_{11}=\frac{1+\nu}{9 E}\left(\frac{4}{1-D_{1}}+\frac{1}{1-D_{2}}+\frac{1}{1-D_{3}}\right) \sigma_{11}+\frac{\sigma_{11}}{9 \tilde{K}}
$$

For tensile loads in $y$ and $z$ directions, the strains $\varepsilon_{22}$ and $\varepsilon_{33}$ can be derived in the same way. These formula lead to the three relations of the damaged Young's moduli,

$$
\begin{aligned}
& \frac{1}{\tilde{E}_{11}}=\frac{1+\nu}{9 E}\left(\frac{3}{1-D_{1}}+\frac{1}{1-D_{1}}+\frac{1}{1-D_{2}}+\frac{1}{1-D_{3}}\right)+\frac{1}{9 \tilde{K}} \\
& \frac{1}{\tilde{E}_{22}}=\frac{1+\nu}{9 E}\left(\frac{3}{1-D_{2}}+\frac{1}{1-D_{1}}+\frac{1}{1-D_{2}}+\frac{1}{1-D_{3}}\right)+\frac{1}{9 \tilde{K}} \\
& \frac{1}{\tilde{E}_{33}}=\frac{1+\nu}{9 E}\left(\frac{3}{1-D_{3}}+\frac{1}{1-D_{1}}+\frac{1}{1-D_{2}}+\frac{1}{1-D_{3}}\right)+\frac{1}{9 \tilde{K}}
\end{aligned}
$$

making the term $\sum_{k} \frac{1}{1-D_{k}}=\frac{1}{1-D_{1}}+\frac{1}{1-D_{2}}+\frac{1}{1-D_{3}}$ appears in each expression. Adding the last three relations gives:

$$
\frac{1}{\tilde{E}_{11}}+\frac{1}{\tilde{E}_{22}}+\frac{1}{\tilde{E}_{33}}=\frac{2(1+\nu)}{3 E}\left(\frac{1}{1-D_{1}}+\frac{1}{1-D_{2}}+\frac{1}{1-D_{3}}\right)+\frac{1}{3 \tilde{K}}
$$

Finally, using relations (18) and (21) leads to the final form:

$$
1-D_{1}=\frac{2(1+\nu)}{E\left(\frac{5}{\tilde{E}_{11}}-\frac{1}{\tilde{E}_{22}}-\frac{1}{\tilde{E}_{33}}-\frac{1}{3 \widetilde{K}}\right)}
$$

One can check that under iso-damage assumption, i.e. $\tilde{E}_{11}=\tilde{E}_{22}=\tilde{E}_{33}$, relation (17) is recovered. 


\section{References}

Bolander, J. E. and S. Saito: 1998, 'Fracture analysis using spring networks with random geometry'. Engineering Fracture Mechanics 61, 569-591.

Chaboche, J. L.: 1978, 'Description thermodynamique et phénoménologique de la visco-plasticité cyclique avec endommagement'. Ph.D. thesis, Université Paris 6.

Chow, C. L. and J. Wang: 1987, 'An anisotropic theory for continuum damage mechanics'. Int. J. Fract. 33, $3-16$.

Cordebois, J. P. and J. P. Sidoroff: 1982, 'Endommagement anisotrope en élasticité et plasticité'. J.M.T.A., Numéro spécial pp. 45-60.

Cundall, P. A. and O. D. L. Strack: 1979, 'A discrete numerical model for granular assemblies'. Géotechnique 29, 47-65.

D'Addetta, G. A.: 2004, 'Discrete models for cohesive frictional materials'. Ph.D. thesis, Stuttgart University.

D'Addetta, G. A., F. Kun, and E. Ramm: 2002, 'On the application of a discrete model to the fracture process of cohesive granular materials'. Granular Matter 4, 77-90.

Delaplace, A. and A. Ibrahimbegovic: 2006, 'Performance of time-stepping schemes for discrete models in fracture dynamic analysis'. International Journal for Numerical Methods in Engineering 65, 1527-1544.

Delaplace, A., S. Roux, and G. Pijaudier-cabot: 1999, 'Failure and scaling properties of a softening interface connected to an elastic block'. International Journal of Fracture 95, 159-174.

Desmorat, R.: 2004, 'Modèle d'endommagement anisotrope avec forte dissymétrie traction/compression'. In: 5ème journées du Regroupement Francophone pour la Recherche et la Formation sur le Béton (RF2B), Liège, Belgium, 5-6 july.

Desmorat, R.: 2006, 'Positivity of intrinsic dissipation of a class of non standard anisotropic damage models'. C.R. Mécanique 334, 587-592.

Desmorat, R., F. Gatuingt, and F. Ragueneau: 2007, 'Nonlocal anisotropic damage model and related computational aspects for quasi-brittle materials'. Eng. Fracture Mechanics 74, 1539-1560.

Halm, D. and A. Dragon: 1998, 'An anisotropic model of damage and frictional sliding for brittle materials'. European Journal of Mechanics, A/Solids 17, 439-60.

Herrmann, H. J. and S. Roux: 1990, Statistical models for the fracture of disordered media. Elsevier Science Publishers, Amsterdam.

Ju, J.: 1989, 'On energy-based coupled elasto-plastic damage theories: constitutive modeling and computational aspects'. Int. J. Solids Structures 25, 803-833.

Kun, F. and H. Herrmann: 1996, 'A study of fragmentation processes using a discrete element method'. Comp. Meth. Appl. Mech. Eng. 7, 3-18.

Ladevèze, P.: 1983, 'On an anisotropic damage theory'. In: Proc. CNRS Int. Coll. 351 Villars-de-Lans, Failure criteria of structured media, J. P. Boehler ed. 1993. pp. 355-363.

Leckie, F. A. and E. T. Onat: 1981, Tensorial nature of damage measuring internal variables, Chapt. Physical Non-Linearities in Structural Analysis, pp. 140-155. J. Hult and J. Lemaitre eds, Springer Berlin.

Lemaitre, J. and R. Desmorat: 2005, Engineering Damage Mechanics : Ductile, Creep, Fatigue and Brittle Failures. Springer.

Lemaitre, J., R. Desmorat, and M. Sauzay: 2000, 'Anisotropic damage law of evolution'. Eur. J. Mech., A/ Solids 19, 187-208.

Linde, P., A. Schulz, and W. Rust: 2006, 'Influence of modelling and solution methods on the FE-simulation of the post-buckling behaviour of stiffened aircraft fuselage panels'. Composite Structures 73, 229-236.

Mazars, J.: 1984, 'Application de la mécanique de l'endommagement au comportement non linéaire et à la rupture du béton de structure'. Ph.D. thesis, Thèse d'Etat Université Paris 6.

Mazars, J., Y. Berthaud, and S. Ramtani: 1990, 'The unilateral behavior of damage concrete'. Eng. Fract. Mech. 35, 629-635.

Moukarzel, C. and H. J. Herrmann: 1992, 'A vectorizable random lattice'. J. Stat. Phys. 68, 911-923.

Murakami, S.: 1988, 'Mechanical modeling of material damage'. J. App. Mech. 55, 280-286.

Papa, E. and A. Taliercio: 1996, 'Anisotropic damage model for the multi-axial static and fatigue behaviour of plain concrete'. Engineering Fracture Mechanics 55, 163-179.

Potyondy, D. O. and P. A. Cundall: 2004, 'A bonded-particle model for rock'. International Journal of Rock Mechanics and Mining Sciences 41(8), 1329-1364. 
Reese, S.: 2006, 'Meso-macro modelling of fibre-reinforced rubber-like composites exhibiting large elastoplastic deformation'. International Journal of Solids and Structures 140, 951-980.

Rots, J. G., S. Invernizzi, and B. Belletti: 2006, 'Saw-tooth softening/stiffening - a stable computational procedure for RC structures'. Computers \& Concrete 3, 213-233.

Schlangen, E. and E. J. Garboczi: 1997, 'Fracture Simulations of Concrete Using Lattice Models: Computational Aspects'. Eng. Fracture Mech. 57(2/3), 319-332.

Van Mier, J. G. M. and M. R. A. Van Vliet: 2003, 'Influence of microstructure of concrete on size/scale effects in tensile fracture'. Engineering Fracture Mechanics 70, 2281-2306.

Van Mier, J. G. M., M. R. A. Van Vliet, and T. K. Wang: 2002, 'Fracture mechanisms in particle composites: statistical aspects in lattice type analysis'. Mech. Mater 34, 705-724.

Wang, J., S. L. Crouch, and S. G. Mogilevskaya: 2006, 'Numerical modeling of the elastic behavior of fiber-reinforced composites with inhomogeneous interphases'. Composites Science and Technology 66, $1-18$.

Yip, M., Z. Li, B.-S. Liao, and J. Bolander: 2006, 'Irregular lattice models of fracture of multiphase particulate materials'. International Journal of Fracture 140, 113-124. 